\title{
Exploiting the Link: Improving Satellite Communication through Higher Elevation Links
}

\author{
Andrew T. Klesh; James W. Cutler ${ }^{\dagger}$
}

\begin{abstract}
Most small satellites operations, including those in the CubeSat community, maximize the duration of single communication passes with ground stations, but, in doing so, do not maximize the total data transferred. In this paper we examine methods to maximize data download by waiting to begin transmission at a non-intuitive high elevation angle. This elevation angle reduces the slant distance and allows the link to be closed at a higher fixed data rate. Though the transmission duration is shorter, the total data downloaded is greater.

We examine this method for a variety of pass configurations and compare it to distributions of passes for known ground stations around the world. The results of this study (analytic and numerical) are presented along with recommendations for strategies for maximizing the amount of data transmitted for a given satellite orbit. These methods rely on the ability to alter the data rate of a radio while on-orbit, enabled through the use of flexible-rate radios.

We expand this study by examining the amount of data transferred for individual groundstations over the course of a year. It is shown that an optimal fixed data rate can be found such that the amount of data downloaded is maximized for the year. Finally, recommendations for radio development are given for the small satellite community.
\end{abstract}

\section{INTRODUCTION}

\section{A. Overview and Motivation}

$\mathrm{T}$ YPICAL small satellite communication links are attempted near where the satellite passes over the local horizon. This has the benefit of maximizing the duration of communication with the satellite, which helps for coordination and operational activity, but restricts achievable data rates. Since most low-cost satellite radios have a single fixed data rate, the radio system must be tuned to communicate at this low elevation, ${ }^{1}$ which often means that only low data rates can be realized.

As more small satellites take on science missions, however, greater amounts of data are generated on orbit. These missions require methods to improve the total data downloaded, but still are limited to fixedrate radio technology. Thus mission designers are faced with limiting data generation in some manner (onboard processing, compression, sampling, etc) to reduce communication requirements or are forced to increase the satellite size to allow for more advanced radios. To date, several interesting science missions have been prevented from using small satellites because of this limitation. ${ }^{2}$

Rather than increase satellite size, in this paper we consider methods to exploit knowledge of the orbital path to increase the total data downloaded while remaining constrained to a fixed-rate radio. These methods allow for the use of current radios (and small satellite structures), but their use is also investigated for larger missions.

\section{B. Literature Review}

Methods to optimize communication links and radio systems have been studied for years and a wide literature is available. Here we focus on specific methods that seek to improve data throughput for small

*Postdoctoral Researcher, Aerospace Engineering, University of Michigan, 1320 Beal Ave, Ann Arbor, MI USA aklesh@umich.edu

${ }^{\dagger}$ Assistant Professor, Aerospace Engineering, University of Michigan, 1320 Beal Ave, Ann Arbor, MI USA jwcutler@umich.edu 
satellite as these are usually the most constrained systems. Generally research has focused on optimizing the satellite communication system, the groundstation or the radio hardware. A basic small satellite (a CubeSat) communication system design is discussed in 5 while the close relationship between the amateur radio community and the CubeSat community is discussed in 3. The characteristics of any communication system are generally described by link budgets. A thorough description can be found in 1, but the amateur radio community also is exhaustive in its description. ${ }^{4}$ Active antennas and antenna design to improve communications have been considered in 6 and 7 , but these are generally difficult to implement on a small satellite. An alternative to the standard hardware radio, software defined radios, have been considered for CubeSats in 8, but has yet to be realized.

A majority of CubeSat architectures use fixed data-rate radios onboard, often restricted to a few selective baud rates $\left(4800,9600,19200,57600\right.$, etc) ${ }^{3}$ though these rates are chosen by the radio manufacturer rather than the designer. In general we will restrict ourselves to a more flexible fixed rate (any integer rate) but will also consider these specific rate choices and their impact upon the mission.

Much of the literature previously discussed describes the design of communication systems. Several works describe the fact that the system must be designed for the longer communication distance when the satellite is located near the horizon. A few mention the existence of a wide link margin between this design and one for a slant distance when the satellite is directly overhead. ${ }^{9}$ The availability and exploitation of link margin is thoroughly discussed in 10. Much of that discussion is similar to the modeling section in this paper. This paper differs from the existing literature in that we examine methods to exploit the link margin by finding a better fixed data rate to utilize. This exploitation provides the capability for satellites in low-Earth-orbit to potentially double the total data downloaded.

\section{Original Contributions and Paper Outline}

In this paper, the problem of improving data download for small spacecraft is discussed analytically and numerically in the context of a maximization problem. The present paper studies this optimization problem and provides the following original contributions:

- An analysis is presented for data rates, data download and duration of link as a function of elevation angle.

- The elevation at which to begin a transfer of the maximum amount of data is shown not to be at the horizon due to large slant distance and small link margin, but generally at a higher elevation.

- Considerations are given to altitude effects as well as implementation for non-LEO missions.

- A method is shown to provide the data rate giving maximum data transferred over the course of a year.

The remainder of the paper is as follows. In Section II, the problem is formulated while communication and orbital pass models are constructed. In Section III methods are shown to exploit the available link margin with additional considerations for the choice of data rate based on satellite pass numbers over the course of a year. Additional subsections provide details on variable data rates and deep-space mission examples. Conclusions are then provided in Section IV. Orbital and spacecraft parameters for the example satellite are provided in Appendix A.

\section{Problem Formulation and Modeling}

In this section, we model the orbital geometry and a simplified link-budget. In doing so, we identify the limitations of fixed data-rate radios and mathematically formulate the problem considered in this paper.

\section{A. Orbit Pass Geometry}

A satellite in circular orbit has its position and velocity defined through the use of the Keplerian elements, $a$, $e, i, \omega, \Omega$ and $\nu$, also known as the semi-major axis, eccentricity, inclination, argument of periapsis, longitude of the ascending node and true anomaly respectively. With these elements we can find the relative position of the satellite with respect to the ground station's latitude and longitude. For the examples used in this paper, the orbital parameters are defined in Appendix A. 
Much of the following analysis is derived from equations found in Reference 1. Consider a ground station located at latitude and longitude $(\hat{\lambda}, L)$. Figure 1 describes the relationship between the various angles and distances describing the satellite position relative to the the center of the Earth and is taken with permission from Reference 1. Figure 2 describes the relationship between the satellites position and the position of the ground station for a pass.

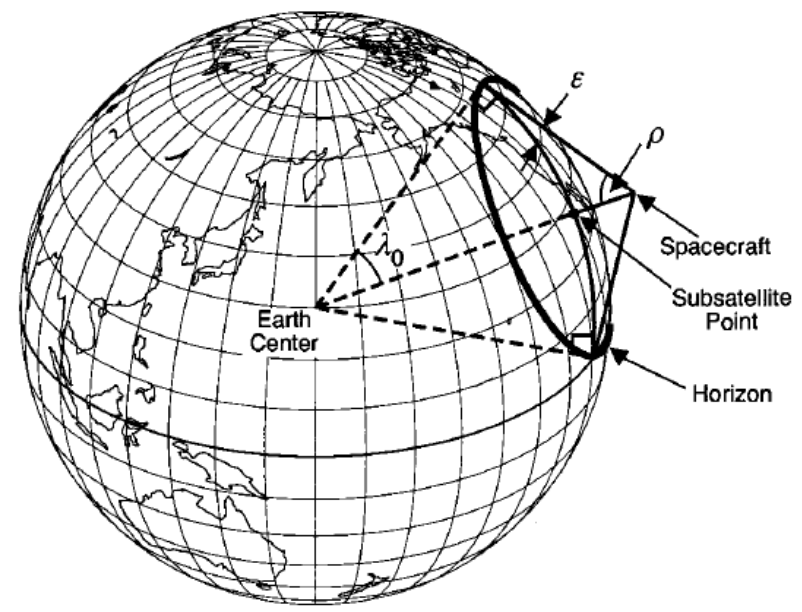

Figure 1. Geometric relationship between an orbiting satellite and a surface groundstation with respect to the center of the Earth. Figure used with permission. ${ }^{1}$

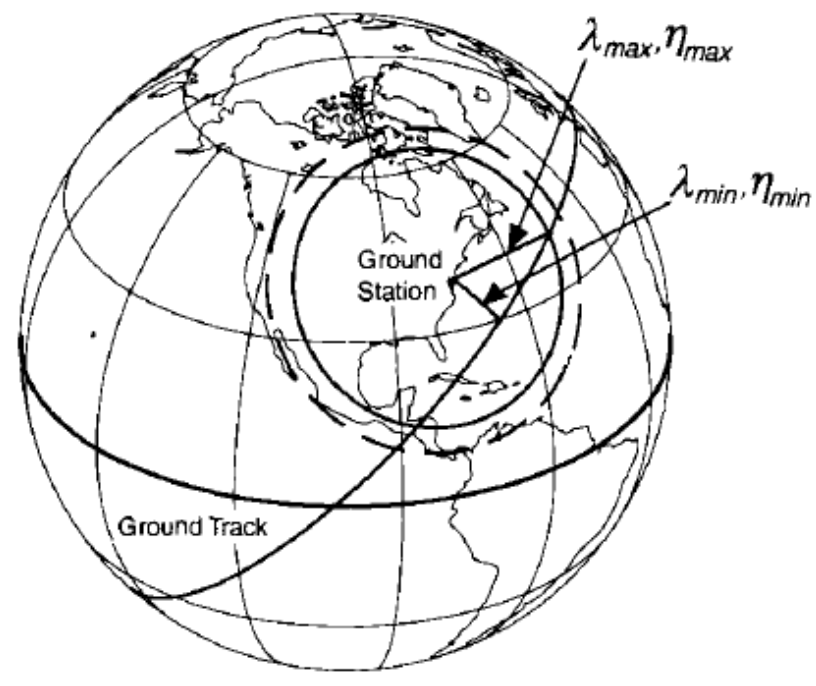

Figure 2. Geometric relationship between a spacecraft and the groundstation during a pass while on a circular orbit. Figure modified and used with permission. ${ }^{1}$

Define $S$ to be the range from the satellite to the ground station. From these figures, it can be seen that $S$ is greatest when the satellite is at the local horizon (when $\lambda=\lambda_{\max }$ ) and least when the satellite passes at the maximum elevation angle (when $\lambda=\lambda_{\min }$ ).

Two expressions for slant distance are provided here based on the geometry presented in Figures 1 and 2:

$$
\begin{aligned}
S & =R_{E}\left[\left(\left(\frac{r+R_{E}}{R_{E}}\right)^{2}-\cos ^{2} \delta_{e}\right)^{1 / 2}-\sin \delta_{e}\right] \\
& =R_{E} \frac{\sin \lambda}{\sin \eta}
\end{aligned}
$$


where $\delta_{e}$ is the elevation angle above the local horizon, $\lambda$ is the Earth central angle and $\eta$ is the nadir angle and can be derived directly from 1 . Of these parameters, $\delta_{e}$ and $S$ are variable while the other parameters remain fixed (for a circular orbit) for each pass. It is possible that over the course of many passes, $\lambda_{\text {max }}$ and $\lambda_{\min }$ will vary (see Section III.A).

\section{B. Pass Time}

The duration of a satellite pass above a ground station can be expressed as:

$$
\begin{aligned}
T & =\frac{P}{180} \arccos \frac{\cos \lambda_{\max }}{\cos \lambda_{\min }}, \\
& =\frac{P}{180} \arccos \frac{90-\delta_{e}-\arcsin \left(\sin \frac{R_{E}}{R_{E}+r} \cos \delta_{e}\right)}{\lambda_{\min }},
\end{aligned}
$$

where $\lambda_{\min }$ can be found as a function of elevation as:

$$
\begin{aligned}
\sin \rho & =\frac{R_{E}}{R_{E}+r}, \\
\sin \eta & =\sin \rho \cos \delta_{e}, \\
\lambda & =90-\delta_{e}-\eta, \\
& \Rightarrow \lambda_{\text {min }}=90-\delta_{e, \max }-\arcsin \left(\frac{R_{E}}{R_{E}+r} \cos \delta_{e, \max }\right) .
\end{aligned}
$$

The pass time will vary over the course of many orbits when the positions of the spacecraft and the groundstation do not exactly align. It directly relates to the total amount of data downloaded for each pass.

\section{Signal-to-Noise Ratio}

The signal-to-noise ratio (SNR) relates all of the parameters in communication systems. A high SNR indicates that a communicated message will likely pass uncorrupted from transmitter to receiver. A low SNR is indicative that the message may be obfuscated. By adjusting the various parameters in a communications system we can observe their effect on the SNR. If the SNR does not exceed a specified value, communications cannot occur.

From Reference 1 an approximate SNR of a communication system is given by the link equation as:

$$
\mathrm{SNR}=\frac{P L_{l} G_{t} L_{s} L_{a} G_{r}}{k T_{s} D_{r}}
$$

where $P$ is the transmitter power, $L_{l}$ is the transmitter-to-antenna line loss, $G_{t}$ is the transmit antenna gain, $L_{s}$ is the space loss, $L_{a}$ is transmission path loss, $G_{r}$ is the receive antenna gain, $k$ is Boltzmann's constant, $T_{s}$ is the system noise temperature and $D_{r}$ is the data rate. For a given orbit, only the $L_{s}$ parameter will vary.

Furthermore, we can expand $L_{s}$ as in Reference 1 as:

$$
L_{s}=\frac{\lambda}{(4 \pi S)^{2}},
$$

where $\lambda$ is the wave-length of the transmitted signal and $S$ is the path length in meters.

By combining and re-arranging these equations, we can examine the relationship between path length and data rate as:

$$
\begin{aligned}
\mathrm{SNR} & =\frac{P L_{l} G_{t} \lambda L_{a} G_{r}}{16 k T_{s} D_{r} \pi^{2} S^{2}} \\
\Rightarrow D_{r} & =\frac{P L_{l} G_{t} \lambda L_{a} G_{r}}{16 k T_{s} \pi^{2} S^{2}[\mathrm{SNR}]}, \\
& =\frac{\alpha}{S^{2}}
\end{aligned}
$$


where $\alpha=\frac{P L_{l} G_{t} \lambda L_{a} G_{r}}{16 k T_{s} \pi^{2}[\mathrm{SNR}]}$ and is approximately constant assuming a fixed SNR as needed for communications and given satellite and orbital parameters for each communication pass. In this paper, path length is directly equivalent to slant distance. It can be seen, therefore, that the available data rate for a given SNR is approximately inversely related to the square of the distance the signal must travel.

To receive data, however, the SNR must be maintained at a high enough value for the message to be detected in spite of noise. The excess SNR above the minimum SNR level for communication is known as the link margin. For instance, a data rate chosen (with associated minimum required SNR) may not be viable for a long slant distance (when the spacecraft is at the horizon) as opposed to a short one (when the spacecraft is overhead).

Ideally in a pass, the link margin is minimized. Excess margin implies that a faster data rate could be used (or more power is being utilized than required), thus more data could be transmitted down. Unfortunately, some margin must be maintained (due to unpredictable effects, such as weather, that affect the noise level), typically about $3 \mathrm{~dB}$. Available link margin can be predicted through the completion of a link budget and achievable data rate through (10).

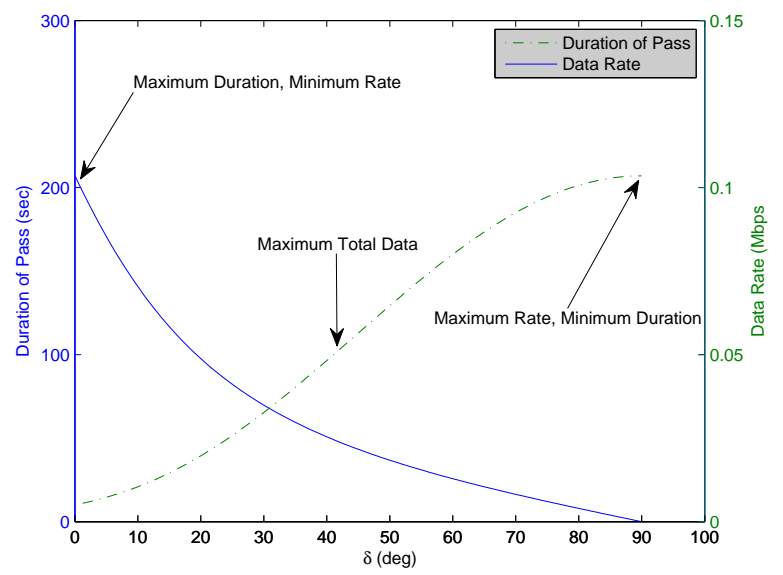

Figure 3. The data rate that can be maintained as a function of elevation and the corresponding pass duration for that data rate. For each elevation where communication begins ( $x$-axis) the corresponding maximum data rate and duration of link can be found ( $y$-axis). Orbit and satellite parameters can be found in Appendix A.

\section{Problem Formulation}

Based on the integrated communications model presented, the problem in this paper is to maximize the total data downloaded for each pass while constrained to transmit at a single fixed data rate, or:

$$
\max _{D_{r}} \int_{t_{0}}^{t_{f}} a D_{r} d t
$$

and subject to the constraint that:

$$
a= \begin{cases}0 & \text { if } \mathrm{SNR}<3 \mathrm{~dB}, \\ 1 & \text { otherwise }\end{cases}
$$

where $a$ indicates the availability of a link. This is equivalent to examining a saturation control problem, where a constant control strategy is needed to maximize a cost function, but it is likely that any control chosen will have regions of zero or inefficient impact on the cost. 


\section{Exploiting Link Margins}

The link budget, as modeled in the presented problem formulation, has excessive link margin for some slant distances that is in excess of what is needed for good communications. In this section, we examine methods to exploit this margin such that total data downloaded can be increased.

\section{A. Constant Data Rates}

The majority of ground stations and satellites attempt to communicate as soon as the satellite passes over the local horizon, i.e., when $\delta_{e}=0$. For a sample satellite, with communication and orbital parameters as in Appendix A, the fixed data rate that can be maintained as a function of elevation is shown in Figure 4.

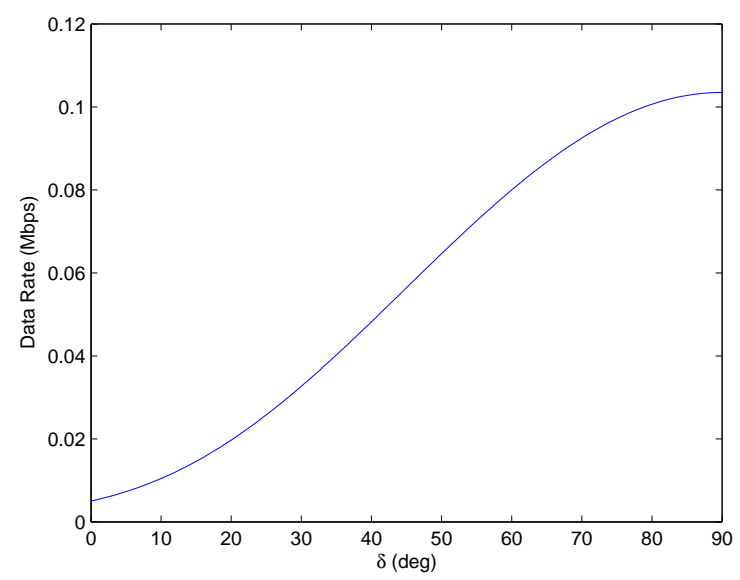

Figure 4. The data rate that can be maintained as a function of elevation, $\delta_{e}$. Here we assume the satellite passes directly over the groundstation. Orbit and satellite parameters can be found in Appendix A. It can be seen that significantly higher data rates can be achieved as the elevation increases, or equivalently, as the slant distance decreases.

The majority of radios used in satellite communications are built for a single data rate. The data rate chosen for the radio is such that the satellite can communicate with the ground station (given antenna and other link budget parameters) at approximately 5 deg above the horizon. While Figure 4 indicates that much higher data rates could be achieved at higher elevations, the radio is constrained to a single rate (i.e., the control is saturated once this elevation is reached and is unfeasible at lower elevations) and is unchangeable after launch.

Consider Figure 3. Here a constant data rate is chosen for each elevation angle and maintained throughout the satellite pass. Higher data rates cannot be maintained for the entire pass and so data is only transferred while the link margin is positive. In the figure, we compare the duration of communication in a pass versus the data rate that can be maintained. It can be seen that high data rates can only be maintained for short periods of time.

In Figure 5 we examine the total data transferred if we were to choose a fixed data rate for a pass and maintain it for the duration of achievable communications. This is equivalent to choosing an elevation angle to begin communications and choosing a data rate such that the link margin in minimized at this elevation. It can be seen that a maximum exists for the amount of data that can be transferred from a satellite in a pass and it is not at the minimum elevation angle for communications.

Analytically we can express the total amount of data transferred as:

$$
\text { Data }=\alpha R_{E}^{2}\left[\left(\left(\frac{r+R_{E}}{R_{E}}\right)^{2}-\cos ^{2} \delta_{e}\right)^{1 / 2}-\sin \delta_{e}\right]^{2} \frac{P}{180} \arccos \frac{90-\delta_{e}-\arcsin \left(\sin \frac{R_{E}}{R_{E}+r} \cos \delta_{e}\right)}{\lambda_{\min }}
$$

which is simply the data rate, held constant for a given elevation angle, multiplied by the duration that the link can be maintained. 
Table 1. The amount of data downloaded, minimum elevation to begin communication and duration of communication link for standard data rates. Note that the maximum data transferred happens during a high speed, short-duration passes, but not the highest speed pass possible.

\begin{tabular}{c|c|c|c}
\hline Baud Rate & Minimum Elevation & Total Duration & Data Downloaded \\
\hline 4800 & $0 \mathrm{deg}$ & $6.9 \mathrm{~min}$ & $1.99 \mathrm{Mb}$ \\
9600 & $9.16 \mathrm{deg}$ & $4.84 \mathrm{~min}$ & $2.79 \mathrm{Mb}$ \\
19200 & $19.48 \mathrm{deg}$ & $3.13 \mathrm{~min}$ & $3.61 \mathrm{Mb}$ \\
57600 & $45 \mathrm{deg}$ & $1.43 \mathrm{~min}$ & $4.94 \mathrm{Mb}$ \\
84000 & $63 \mathrm{deg}$ & $22.76 \mathrm{sec}$ & $2.91 \mathrm{Mb}$ \\
115200 & $\mathrm{~N} / \mathrm{A}$ & $\mathrm{N} / \mathrm{A}$ & $\mathrm{N} / \mathrm{A}$ \\
\hline
\end{tabular}

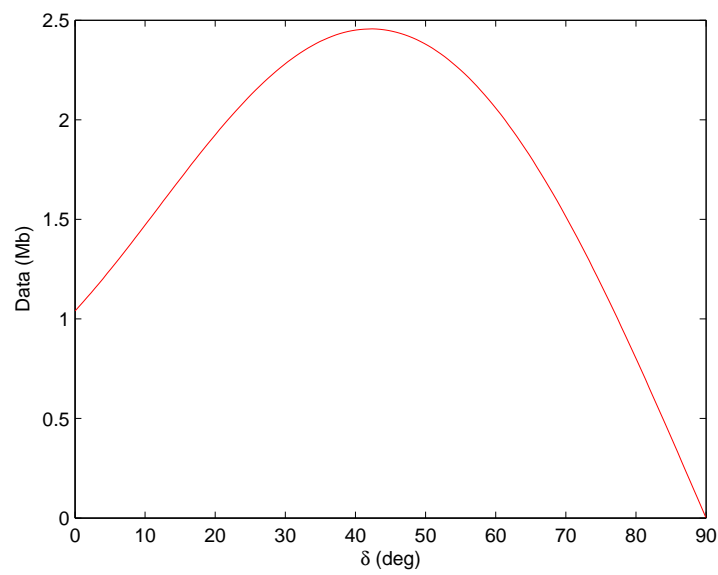

Figure 5. The total data downloaded as a function of elevation (data rate). For each elevation where communication begins ( $x$-axis) the corresponding maximum data transferred can be found ( $y$-axis). Note that a maximum exists at approximately $42 \mathrm{deg}$ elevation. Orbit and satellite parameters can be found in Appendix A.

Consider the standard bauds $(4800,9600,19200$, etc) which are often the selectable data rates for common radios. At these rates, we can calculate the total amount of data downloaded as in Table 1.

We can numerically solve for the point of maximum data for each pass (an analytic solution is generally difficult). If we consider that the satellite might not pass directly over the ground station, we can consider a family of passes and find the elevation angles at which to begin communication where the most data can be transferred. Figures 6 and 7 indicate these angles for our sample satellite.

\section{B. Variable Data Rates}

The ideal situation would be for a flexible radio to change data rates as needed to always maximize the amount of data transferred while respecting communication link constraints. Often this requires the use of software-defined radios (where many parameters are flexible), or the ability for the data-rate switching to happen quickly. Reference 10 discusses this approach for CubeSats and the use of changing fixed-data rates. Unfortunately this requires both the groundstation and spacecraft to remain in sync, so some overhead or pre-arrangement is required for this to work. An alternative method is the use of clock-less signals (or rate-less signals), which supplies a clocking signal along side the data signal. ${ }^{11}$ With the common small radio unable to change data rate frequently enough to exploit variable data rates and rate-less radios not yet available for small satellites, these methods must be reserved for future use. 


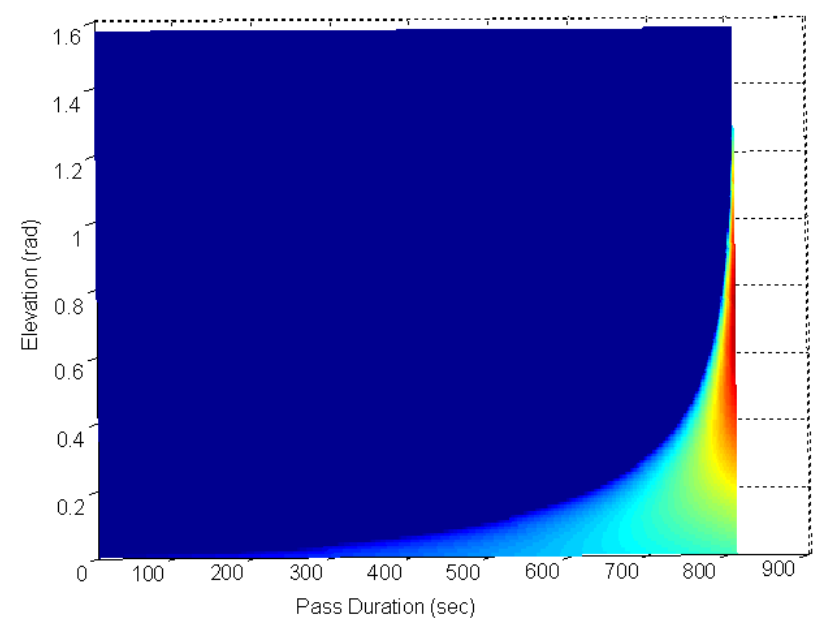

Figure 6. The total amount of data downloaded as a function of the elevation angle to begin communications and the pass duration. The more red the data point is, the more information that can be downloaded.

\section{Altitude Effects}

As the semi-major axis of the circular orbit is increased, the gain in data transferred by optimizing the data rate as before decreases. By approximately 10,000 km altitude over the Earth, almost no gain is provided through this method. This is due to the fact that over the pass, the slant distance, from horizon to overhead, changes very little as the altitude increases. We examine the amount by which the slant distance changes (and by extention through Equation 16, the data rate that we can maintain) in Figure 8.

Consider the following analytic proof. The slant distance, given by Equation 1, is defined at the horizon and directly overhead the ground station respectively as:

$$
\begin{aligned}
& S_{\mathrm{H}}=R_{e}\left[\left(\left(\frac{r+R_{e}}{R_{e}}\right)^{2}-1\right)^{1 / 2}\right], \\
& S_{\mathrm{O}}=R_{e}\left[\left(\left(\frac{r+R_{e}}{R_{e}}\right)-1\right)\right] .
\end{aligned}
$$

If we compare a ratio of these two slant distances, $\frac{S_{\mathrm{H}}}{S_{\mathrm{O}}}$, we can simplify as:

$$
\text { Distance Ratio }=\sqrt{1+2 \frac{R_{e}}{r}} .
$$

As $r \rightarrow \infty$, the so-called Distance Ratio approaches 1. Therefore as altitude increases, the change in slant distance decreases, reducing the total link margin available to exploit.

This ratio also depends strongly on the radius of the object being orbited. Consider the asteroid Itokawa, the target of the recently completed Hayabusa mission. The small Minerva rover was designed to be deployed to the surface while the Hayabusa spacecraft remained in orbit overhead. Though irregularly shaped, we will assume that Minerva, upon landing, would operate on the surface of a bounding sphere with radius $R_{A}$ co-located with the center of mass.

Minerva carried a small radio operating at $9600 \mathrm{bps}$ with a range of $20 \mathrm{~km} \cdot{ }^{12}$ We will assume $R_{A}=0.27 \mathrm{~km}$ (the largest dimension of Itokawa ${ }^{13}$ ). If remains in a safe circular orbit of approximately $1 \mathrm{~km}$ altitude, ${ }^{13}$ we can find the ratio of maximum to minimum slant distances to be 1.24 leading to a corresponding potential total data gain if link dynamics are taken into account.

If we instead examine actual events, where Hayabusa remained at its home position ( $7 \mathrm{~km}$ altitude) for a majority of its time near Itokawa, we can see almost no gain can be achieved with these methods.

Since asteroid mission spacecraft usually are equipped with light-weight and robust equipment (often fixed-data-rate radios), they might well benefit from the methods proposed in this paper. 


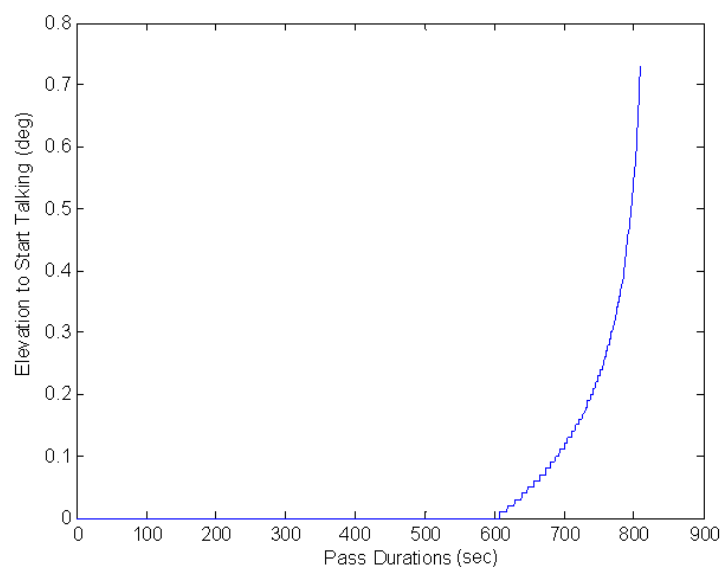

Figure 7. The elevation at which a maximum amount of data is downloaded as a function of pass duration. Note that for short passes, it is better to begin communication as early in the pass as possible as the satellite does not achieve a very high elevation during the pass.

\section{Global Passes}

Over the course of a year, a spacecraft will pass a ground station with a variety of pass durations and slant distances. We can examine the number of passes a satellite makes of a ground station at different latitudes and pass durations in the form of a histogram as in figure 9 (Note that these figures are numerically derived with a coarse grid leading to some numerical discrepencies).

Consider a particular ground station, at Ann Arbor, MI, so that we can look closer as in figure 10.

Given the data rate corresponding to the most data downloaded for each pass, we can maximize the total data downloaded for an entire year to design an optimal fixed data rate. This global rate does not necessarily match the local, single pass rate. This is illustrated in figure 11.

Future work will investigate analytic and numerical tools to find the optimal ground station locations to use for a given orbit based on this histogram information.

\section{Conclusions}

This paper has investigated methods to exploit the communication link of small satellites that are required to use fixed data rates. By choosing an appropriate data rate, total data downloaded can be maximized. This method primarily works for LEO missions and loses its effectiveness as a function of altitude. In conjunction with global pass coverage analysis, the methods presented can help designers plan for optimal placement of groundstations and the optimal fixed data rate to use for the ground stations planned.

The concepts illustrated here presented here alter the normal mission planning strategy for small spacecraft communication links. Previous methods have been based on the idea that more time provides more data. In this paper we have shown that this is not always optimal. Future work will investigate tools making use of these methods for communication link design. It will also examine flexible radios that can change their data rate and so follow an optimal data rate curve to maximize data downloaded.

\section{Acknowledgements}

The work completed in this paper was supported in part by NSF Grant 0838046. The authors would also like to acknowledge the many useful suggestions given by various members of the Radio Aurora Explorer (RAX) team at the University of Michigan. 


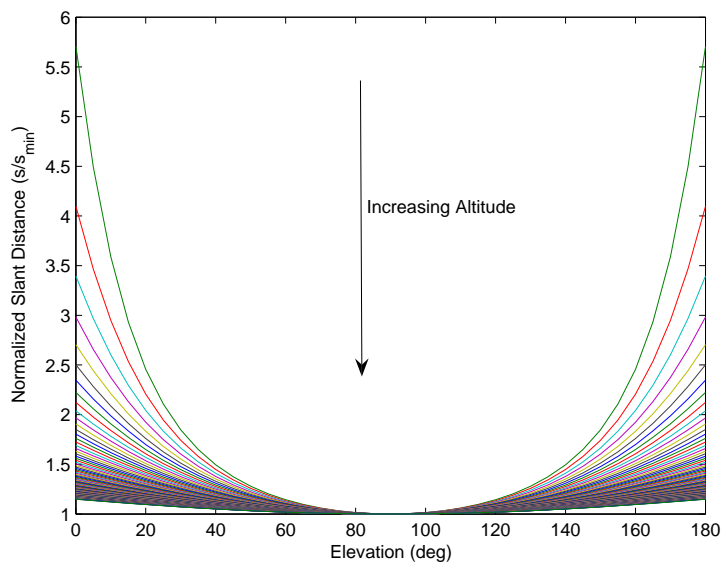

Figure 8. Comparison of slant distance changes as a function of pass elevation. As slant distance is inversely proportional to data rate, a significant change in slant distance over a satellite pass indicates that a large link margin might exist that can be exploited to achieve higher data throughput.

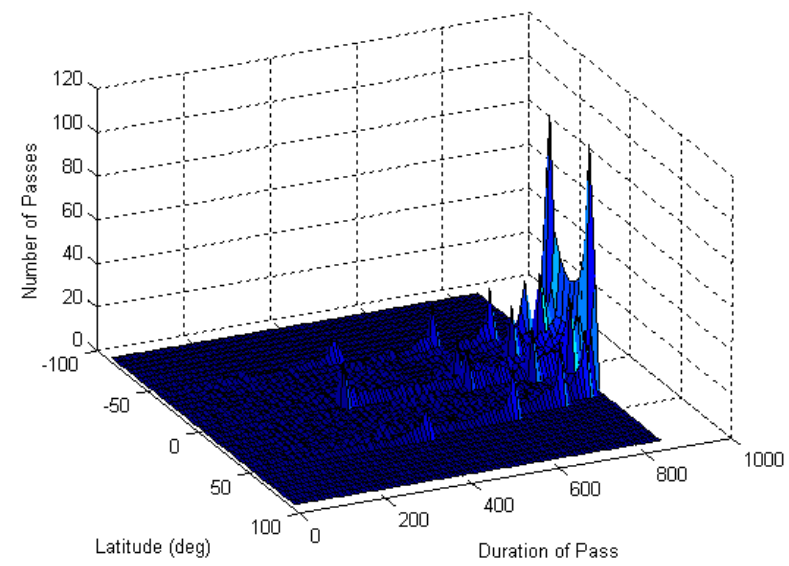

Figure 9. A histogram of pass durations based on latitude over the course of a year. 


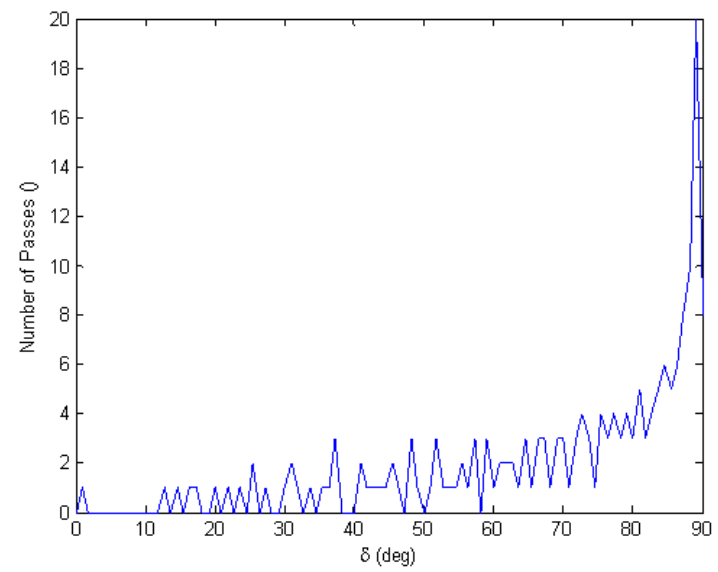

Figure 10. A histogram of pass durations based on latitude over the course of a year. Spacecraft and orbit parameters can be found in Appendix A. Note that the longest passes occur around 50 deg elevation.

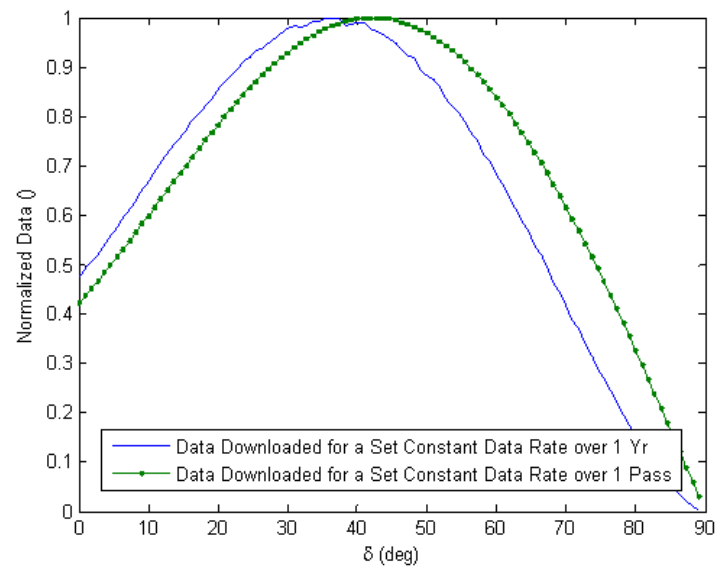

Figure 11. Comparison of data rates between global and single passes. 


\section{Appendix A: Satellite and Orbit Parameters}

The example spacecraft used throughout this paper has the following properties:

Table 2. Spacecraft Parameters

\begin{tabular}{lccc}
\hline Transmit Antenna Gain & $G_{t}$ & 0.1566 & $d B$ \\
Frequency & $f$ & 440 & $\mathrm{MHz}$ \\
Receive Antenna Gain & $G_{a}$ & 0.711 & $\mathrm{~dB}$ \\
Power & $P_{w}$ & 0.7 & $\mathrm{~W}$ \\
\hline
\end{tabular}

The example orbit used throughout this paper has the following properties:

Table 3. Orbit Parameters

\begin{tabular}{lccc}
\hline Semi-Major Axis & $a$ & 7028.15 & $\mathrm{~km}$ \\
Eccentricity & $e$ & 0 & \\
Inclination & $i$ & 72 & $\mathrm{deg}$ \\
\hline
\end{tabular}

\section{References}

\footnotetext{
${ }^{1}$ Wertz, J. and Larson, W., "Space mission analysis and design," Space mission analysis and design, by Wertz, James Richard.; Larson, Wiley J. El Segundo, Calif.: Microcosm Press; Dordrecht; Boston: Kluwer, c1999. Space technology library, Vol. 8, 1999.

${ }^{2}$ Lein, J., "Assessing the applicability of satellite imagery for environmental monitoring and regulatory compliance assessment," International Journal of Environmental Technology and Management, Vol. 4, No. 1, 2004, pp. 157-173.

${ }^{3}$ Klofas, B., Anderson, J. and Leveque, K., "A Survey of CubeSat Communication Systems," California Polytechnic State University and SRI International Technical Report, 2008. 2009.

${ }^{4}$ Maral, G., Bousquet, M. and Sun, Z. "Satellite Communications Systems: Systems, Techniques and Techology," Wiley, 2009.

${ }^{5}$ Schroer, M., Reader, S., and Smith, T., "NPS-SCAT: A CubeSat Communications System Design, Test, and Integration,"

${ }^{6}$ Fujishige, T., Ohta, A., Tamamoto, M., Goshi, D., Murakami, B., Akagi, J., and Shiroma, W., "Active Antennas for CubeSat Applications," 16th Annual AIAA/Utah State University Conference on Small Satellites, Logan, UT, paper SSC02, Vol. 2, 2002.

${ }^{7}$ Tamamoto, M., "Active Antennas and UHF Antennas for CubeSat Applications," .

${ }^{8}$ Corrado, B., Ebel, W., and Jayaram, S., "Cubesat Software Defined Radio Project," 26th International Communications Satellite Systems Conference(ICSSC), American Institute of Aeronautics and Astronautics, 1801 Alexander Bell Drive, Suite 500, Reston, VA, 20191-4344, USA,, 2008.

${ }^{9}$ Davarian, F., Shambayati, S. and Slobin, S., "Deep Space Ka-Band Link Management and Mars Reconnaissance Orbiter: Long-Term Weather Statistics Versus Forecasting," Proceedings of the IEEE, Vol. 92, No. 12, 2004.

${ }^{10}$ Palmer, J. and Caffrey, M., "Exploiting Link Dynamics in LEO-to-Ground Communications," Proceedings of the 2009 Small Sat Conference, 2009.

${ }^{11}$ Erez, U., Trott, M., and Wornell, G., "Rateless Coding for Gaussian Channels," IEEE Transactions of Information Theory, 2007.

${ }^{12}$ Yoshimitsu, T., Kubota, T., Nakatani, I., Adachi, T., and Saito, H., "Micro-hopping Robot for Asteroid Exploration," Acta Astronautica, Vol. 52, pp.441-446, 2003.

${ }^{13}$ Scherees, D., Broschart, S., Ostro, S., and Benner, L., "The Dynamical Environment About Asteroid 25143 Itokawa: Target of the Hayabusa Mission," AIAA/AAS Astrodynamics Specialist Conference and Exhibit, 2004.
} 